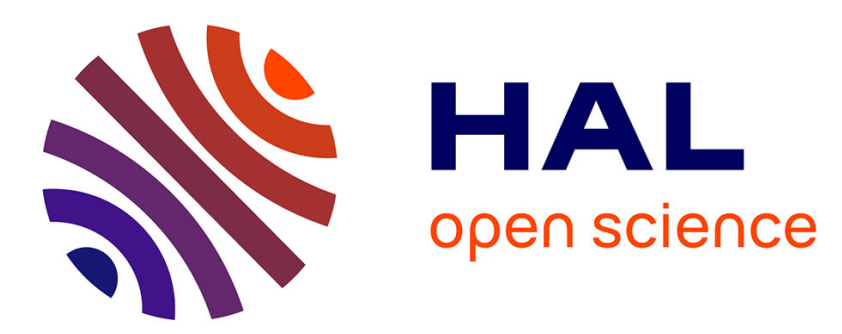

\title{
A model of a syntrophic relationship between two microbial species in a chemostat including maintenance
}

T. Sari, Jérôme Harmand

\section{To cite this version:}

T. Sari, Jérôme Harmand. A model of a syntrophic relationship between two microbial species in a chemostat including maintenance. Mathematical Biosciences, 2016, 275, pp.1-9. 10.1016/j.mbs.2016.02.008 . hal-01541383

\section{HAL Id: hal-01541383 \\ https://hal.science/hal-01541383}

Submitted on 19 Jun 2017

HAL is a multi-disciplinary open access archive for the deposit and dissemination of scientific research documents, whether they are published or not. The documents may come from teaching and research institutions in France or abroad, or from public or private research centers.
L'archive ouverte pluridisciplinaire HAL, est destinée au dépôt et à la diffusion de documents scientifiques de niveau recherche, publiés ou non, émanant des établissements d'enseignement et de recherche français ou étrangers, des laboratoires publics ou privés. 


\section{Abstract}

Many microbial ecosystems can be seen as microbial 'food chains' where the different reaction steps can be seen as such: the waste products of the organisms at a given reaction step are consumed by organisms at the next reaction step. In the present paper we study a model of a two-step biological reaction with feedback inhibition, which was recently presented as a reduced and simplified version of the anaerobic digestion model ADM1 of the International Water Association (IWA). It is known that in the absence of maintenance (or decay) the microbial 'food chain' is stable. In a previous study, using a purely numerical approach and ADM1 consensus parameter values, it was shown that the model remains stable when decay terms are added. However, the authors could not prove in full generality that it remains true for other parameter values. In this paper we prove that introducing decay in the model preserves stability whatever its parameters values are and for a wide range of kinetics.

9 Keywords: Microbial ecosystems, Syntrophic relationship, Maintenance,

$11 \quad 2010$ MSC: 34D20, 92D25, 92C45

\section{Introduction}

Two-step models are commonly used to describe microbial systems, which take the form of a cascade of two biological reactions where one substrate $S_{0}$

\footnotetext{
* Corresponding author

Email addresses: tewfik.sari@irstea.fr (Tewfik Sari), Preprint submitted to Mathematical Biosciences
} 
is consumed by one microorganism $X_{0}$ to produce a product $S_{1}$ that serves as the main limiting substrate for a second microorganism $X_{1}$ as schematically represented by the following reaction scheme:

$$
S_{0} \stackrel{f_{0}}{\longrightarrow} X_{0}+S_{1}, \quad S_{1} \stackrel{f_{1}}{\longrightarrow} X_{1}
$$

where $f_{0}$ and $f_{1}$ are the nutrient uptake functions that may depend on several substrates. The substrate and biomass concentrations in this two-step model evolve according to the four-dimensional dynamical system of ODEs

$\left\{\begin{aligned} \frac{d S_{0}}{d t} & =D\left(S_{0}^{i n}-S_{0}\right)-f_{0}(\cdot) X_{0} \\ \frac{d X_{0}}{d t} & =-\alpha D X_{0}+Y_{0} f_{0}(\cdot) X_{0}-a_{0} X_{0} \\ \frac{d S_{1}}{d t} & =D\left(S_{1}^{i n}-S_{1}\right)+\beta\left(1-Y_{0}\right) f_{0}(\cdot) X_{0}-f_{1}(\cdot) X_{1} \\ \frac{d X_{1}}{d t} & =-\alpha D X_{1}+Y_{1} f_{1}(\cdot) X_{1}-a_{1} X_{1}\end{aligned}\right.$

where $Y_{0} \leq 1$ and $Y_{1} \leq 1$ are yield coefficients and $\beta \leq 1$ denotes an appropriate constant. More precisely the terms $Y_{0} f_{0}(\cdot) X_{0}$ and $Y_{1} f_{1}(\cdot) X_{1}$ in the second and fourth equations are the flux towards the biomasses $X_{0}$ and $X_{1}$ respectively. The terms $\left(1-Y_{0}\right) f_{0}(\cdot) X_{0}$ and $\left(1-Y_{1}\right) f_{1}(\cdot) X_{1}$ represent the flux towards other products that may or not be included in the model according to their interactions with the dynamical variables. For instance, in the third equation, $\beta\left(1-Y_{0}\right) f_{0}(\cdot) X_{0}$ is the part which goes to substrate $S_{1}$ and the rest goes to other products which are left out in our model. Here, it is assumed that the units of the state variable are well chosen. Mathematically we can perform a rescaling of the variables, that is to say we change the units of the state variables, such that the constant parameters $Y_{0}, Y_{1}$ and $Y_{2}=\beta\left(1-Y_{0}\right)$ are fixed to 1 , see (8) and model (9) below. For a typical example, see model (21) in Section 6.

Substrate $S_{0}$ and $S_{1}$ are introduced with an input concentration $S_{0}^{i n}$ and $S_{1}^{i n}$ respectively, and at dilution rate $D$. Depending on the technology used to confine the reactions, the coefficient $\alpha \leq 1$ is not necessarily equal to 1 and $1-\alpha$ represents the proportion of biomass which is retained in the reactor. This model includes the maintenance (or decay) terms $a_{0}$ and $a_{1}$. Maintenance, in 
its most general assertion, is the consumption of energy for all processes other than growth: it is modelled either by adding a negative term on the substrate dynamic without associating it to growth or by considering a decay term on the biomass dynamics, as in (1). For more information about the modelling of maintenance, the reader is referred to [16]. These models present the advantage of being complex enough to capture important process properties while being simple enough to be mathematically studied.

When the growth function $f_{0}$ depends only on the substrate $S_{0}$ and the growth function $f_{1}$ depends only on the substrate $S_{1}$, that is

$$
f_{0}(\cdot)=f_{0}\left(S_{0}\right), \quad f_{1}(\cdot)=f_{1}\left(S_{1}\right),
$$

the system is known as commensalistic: one species grows on the product of another one $[18,22]$. The system has a cascade structure: solve the first and second equations for $S_{0}, X_{0}$, and then use this result is the remaining equations to find $S_{1}, X_{1}$. Consequently $S_{0}$ and $X_{0}$ are the same in pure and mixed culture experiments. The number of steady-states and their stability as a function of model inputs and parameters may be investigated [4, 5, 21].

When $f_{0}$ depends on both substrates $S_{0}$ and $S_{1}$ and $f_{1}$ depends only on $S_{1}$, that is

$$
f_{0}(\cdot)=f_{0}\left(S_{0}, S_{1}\right), \quad f_{1}(\cdot)=f_{1}\left(S_{1}\right)
$$

the system is known as syntrophic. For instance if the first organism is inhibited by high concentrations of the product $S_{1}$, the extent to which the substrate $S_{0}$ is degraded by the organism $X_{0}$ depends on the efficiency of the removal of the product $S_{1}$ by the bacteria $X_{1}$. The mathematical analysis of such model is more delicate than commensalistic models, see for instance $[8,14,15,30]$ and the more recent papers $[10,11,19,26,31]$.

A model of a two-tiered microbial 'food chain' with feedback inhibition, which encapsulates the essence of the anaerobic digestion process was recently proposed [31]. Anaerobic digestion is a biological process that converts organic matter into a gaseous mixture composed mainly of methane and carbon dioxide through the action of a complex bacterial and archaeal ecosystem. It is 
often used for the treatment of concentrated wastewaters or to convert the excess sludge produced in wastewater treatment plants into more stable products $[17,23]$. One of its advantages is that the methane produced can be used profitably as a source of energy. It is usually considered that a number of metabolic groups of microorganisms are involved sequentially in several serial and parallel conversion steps to finally produce methane and carbon dioxide. The Anaerobic Digestion Model No. 1 (ADM1) of the IWA Task Group for Mathematical Modelling of Anaerobic Digestion Processes [3, 12] is too complex to permit mathematical analysis of its nonlinear dynamics and only numerical investigations are available [7].

The model of $\mathrm{Xu}$ et al. [31] includes maintenance terms and considers the syntrophic associations between propionate degraders and methanogens. The authors did not show that the non-trivial steady-state is necessarily stable. In addition, simulation results, with the ADM1 consensus values, indicate that the positive steady-state is always stable whenever it exists. For the operators of anaerobic wastewater treatment systems the results of $\mathrm{Xu}$ et al. [31] show that the syntrophic associations between propionate degraders and methanogens are inherently stable under realistic environmental conditions. However, the possibility of an unstable positive steady-state was not excluded for other parameter values and the title of [31], Maintenance affects the stability of a two-tiered microbial 'food chain'? left unanswered the question of the effects of maintenance from a more general viewpoint. In the present paper, we show that for any values of the parameters the positive steady-state is stable as long as it exists, that is to say, maintenance does not affect the stability of the considered two-tiered microbial 'food chain', see [20].

The paper is organized as follows. In Section 2, we review the different twostep models that have been proposed in the literature and we recall what useful informations were obtained for applications from their qualitative mathematical analysis. In Section 3 we present the hypothesis on $(1,3)$. In Section 4 we give the description of the steady-state and their stability. In Section 5 we describe the operating diagram. In Section 6 we apply our results to the model of $\mathrm{Xu}$ et 
al. [31] and we give an answer to open questions on the stability of the positive steady-state of their model. Concluding remarks are given in Section 7. The technical proofs of the results are given in the Appendix.

\section{Commensalism, mutualism and syntrophy}

The different analyses of the class of models (1) available in the literature essentially differ on the way the growth rate functions are characterized and whether a specific input for $S_{1}$ or a coefficient $\alpha$ in the dilution rate of the biomass is considered or not. In most cases, the models used are not generic in the sense either model parameters are fixed or the growth functions are predefined (Monod, Haldane, etc). For details and informations on the various models considered in the existing literature the reader can consult Table $2 \mathrm{E}$ and Table 3 in the review paper [27]. Here we give a short review of the main results which are related to our work.

Following Stephanopoulos [22] we say that 'Two populations of microorganisms which grow in a mixed culture and interact in such a way that one population (the commensal population) depends for its growth on the other population and thus benefits from the interaction while the other population (the host) is not affected by the growth of the commensal population constitutes an example of commensalism'. Reilly [18] was the first to propose a mathematical study of a pure commensalistic model $(1,2)$, with $a_{0}=a_{1}=0$ and $\alpha=1$. He was interested in explaining surprising oscillations observed within the course of an experiment realized in making Saccharomyces carlsbergensis growing on fructose produced by Acetobacter suboxyduns from mannitol. In particular, he established theoretical conditions involving a feedback from the yeast to the bacteria. In this study, explicit growth functions modelling the proposed feedback were used.

An important contribution on the modelling of anaerobic digestion as a commensalistic system is the model by Bernard et al. [5]. The authors considered a Monod function for $f_{0}$ and a Haldane function for $f_{1}$. Sbarciog et al. [21] studied this model for $\alpha=1$ while the interesting case where $0<\alpha<1$ and 
where growth functions were characterized by qualitative properties was studied by Benyahia et al. [4]. Prior to these investigations, and regarding the potential of anaerobic systems to produce renewable energy, the study of these models were particularly important for optimizing anaerobic digestion, notably through the synthesis of state observers and control feedback laws (cf. for instance [1,2]).

Another fundamental ecological interactions which can be modelled by twostep reaction models with two microorganisms are mutualism and syntrophy. Mutualism is defined as a situation where two organisms cooperate typically in producing mutually the substrate necessary to the growth of the other [9]. A syntrophic relationship between two organisms refers to growth functions of the form (3) where the species exhibit mutualism but where, in contrast to what happens in a purely symbiotic relationship, one of the species can grow without the other. Important results of these studies were conditions under which a stable coexistence may occur. Wilkinson et al. [30] studied the interactions in a mixed bacterial population growing on methane. They considered the case of growth functions of the form

$$
f_{0}\left(S_{0}, S_{1}\right)=\frac{m_{0} S_{0}}{K_{0}+S_{0}} \frac{1}{1+S_{1} / L_{1}}, \quad f_{1}\left(S_{1}\right)=\frac{m_{1} S_{1}}{K_{1}+S_{1}}
$$

where $S_{0}$ and $S_{1}$ are the dissolved oxygen and methanol concentrations respectively. The parameters $m_{i}$ and $K_{i}, i=1,2$, are the classical Monod (or Michaelis-Menten) constants and $L_{1}$ is the methanol inhibition constant [see 30, Formulas (1) and (2) and Table V].

Kreikenbohm and Bohl [14] considered the case where $f_{1}$ is a Monod function and the growth function $f_{0}$ takes the form

$$
f_{0}\left(S_{0}, S_{1}\right)=\left\{\begin{array}{lll}
\frac{m_{0}\left(S_{0}-S 1 / L\right)}{K_{0}+S_{0}+K_{1} S_{1}} & \text { if } & S_{0}-S 1 / L>0 \\
0 & \text { otherwise }
\end{array}\right.
$$

In this case, $m_{0}$ and $K_{0}$ are the classical Michaelis-Menten constants and $K_{1}$ acts as an inhibition constant related to the negative influence of the substrate $S_{1}$ on its own production. Moreover, the first organism is unable to grow unless the quotient $S_{1} / S_{0}$ is small enough, say, $S_{1}<L S_{0}$, where $L$ denotes an appropriate constant [see 14, Formulas (6a) and (7)]. 
Burchard [8] extended the results of $[14,30]$ to a large class of more generic growth functions, including the special cases (4) and (5). He highlighted conditions under which there is persistence or extinction. El Hajji et al. [10], motivated by the analysis of the main studied steps of the anaerobic digestion where $H_{2}$-producing acetogens are associated to $H_{2}$-utilizing bacteria, considered the general case where the growth functions (3), satisfy the following properties:

$$
\frac{\partial f_{0}}{\partial S_{0}}>0, \quad \frac{\partial f_{0}}{\partial S_{1}}<0, \quad \frac{d f_{1}}{d S_{1}}>0
$$

Another extension was considered by Kreikenbohm and Bohl [15], which considered the case where $S_{0}$ appears also in $f_{1}(\cdot)$ :

$$
f_{1}\left(S_{0}, S_{1}\right)=\frac{m_{1} S_{1}}{K_{1}+S_{1}} \frac{1}{1+S_{0} / L_{0}}
$$

Here, $m_{1}$ and $K_{1}$ are Michaelis-Menten-type constants, and $L_{0}$ is an inhibition constant which represents the negative effect of $S_{0}$ on the growth of $x_{1}$. The mathematical analysis of this model showed the occurrence of bistability that cannot be observed when $f_{1}(\cdot)$ depends only on $S_{0}$. Sari et al. [19] considered the general situation of a growth function $f_{1}(\cdot)=f_{1}\left(S_{0}, S_{1}\right)$, which is increasing in $S_{1}$ and decreasing in $S_{0}$ and showed, in contrast with the case where $f_{1}(\cdot)=$ $f_{1}\left(S_{1}\right)$ depends only on $S_{1}$, that a multiplicity of positive equilibria can occur. This work was motivated by the study of the influence of the presence of an input term into the dynamics of $S_{1}$ again and by the consideration of more general forms for growth rate functions to investigate the association of $H_{2}$-producing acetogens and $H_{2}$-utilizing bacteria. Other models for which $f_{0}(\cdot)=f_{0}\left(S_{0}, S_{1}\right)$ and $f_{1}(\cdot)=f_{1}\left(S_{0}, S_{1}\right)$, exhibiting the multiplicity of positive equilibria can be found in $[26]$.

All these studies do not include maintenance terms. This short review of the existing literature shows that under conditions like (6) and without maintenance terms $\left(a_{0}=a_{1}=0\right)$, the positive steady-state is unique and stable, if its exists $[8,10,14,30]$. On the other hand as soon as $f_{1}(\cdot)=f_{1}\left(S_{0}, S_{1}\right)$ may depend on $S_{0}$ then instability of the positive steady-state can occur $[15,19,26]$.

To the best of our knowledge, $\mathrm{Xu}$ et al. [31] were the first to consider the 
effects of maintenance terms in $(1,3)$, in the particular case of the growth functions (4), and $S_{1}^{i n}=0, \alpha=1$. As mentioned in the introduction these authors were not able to show that the positive steady-state is stable if it exists. In the present paper we will consider the general case $(1,3)$ where growth functions satisfy (6) and with maintenance terms $\left(a_{0}>0, a_{1}>0\right)$ and $S_{1}^{i n}=0$, $\alpha=1$. We will prove that the positive steady-state is stable whenever it exists. Therefore, in this paper we generalize [31] by allowing a larger class of growth functions, we generalize [30] by allowing a larger class of growth functions and maintenance terms, and we generalize [10] by allowing maintenance terms. For the applications our results show that the syntrophic associations between propionate degraders and methanogens are inherently stable for a wide range of kinetics and whatever the parameters values are, not only for the kinetics (4) and with the ADM1 consensus values of parameters as shown in [31].

An important and interesting extension should be mentioned here: Weedermann et al. [28] proposed an 8-dimensional mathematical model, which includes syntrophy and inhibition, both mechanisms considered by Bernard et al. [5] and by El Hajji et al. [10]. The effects of maintenance terms are considered by Weedermann et al. [29].

\section{The model}

In this paper, we study the model $(1,3)$ with $\alpha=1$ and $S_{1}^{\text {in }}=0$. We use the notation $Y_{2}=\beta\left(1-Y_{0}\right)$. We obtain the following system

$200 \quad\left\{\begin{aligned} \frac{d S_{0}}{d t} & =D\left(S_{0}^{i n}-S_{0}\right)-f_{0}\left(S_{0}, S_{1}\right) X_{0} \\ \frac{d X_{0}}{d t} & =-D X_{0}+Y_{0} f_{0}\left(S_{0}, S_{1}\right) X_{0}-a_{0} X_{0} \\ \frac{d S_{1}}{d t} & =-D S_{1}+Y_{2} f_{0}\left(S_{0}, S_{1}\right) X_{0}-f_{1}\left(S_{1}\right) X_{1} \\ \frac{d X_{1}}{d t} & =-D X_{1}+Y_{1} f_{1}\left(S_{1}\right) X_{1}-a_{1} X_{1}\end{aligned}\right.$

Notice that we do not assume any specific analytical expression for the growth and inhibition functions. Our analysis will use only the following general assumptions for the growth functions $f_{0}\left(S_{0}, S_{1}\right)$ and $f_{1}\left(S_{1}\right)$ : 
A1 For all $S_{0}>0$ and $S_{1} \geq 0, f_{0}\left(S_{0}, S_{1}\right)>0$ and $f_{0}\left(0, S_{1}\right)=0$.

A2 For all $S_{1}>0, f_{1}\left(S_{1}\right)>0$ and $f_{1}(0)=0$.

A3 For all $S_{0}>0$ and $S_{1}>0, \frac{\partial f_{0}}{\partial S_{0}}\left(S_{0}, S_{1}\right)>0$ and $\frac{\partial f_{0}}{\partial S_{1}}\left(S_{0}, S_{1}\right)<0$.

A4 For all $S_{1}>0, \frac{d f_{1}}{d S_{1}}\left(S_{1}\right)>0$.

Hypothesis A1 signifies that no growth can take place for species $X_{0}$ without the substrate $S_{0}$. Hypothesis $\mathbf{A} \mathbf{1}$ means that the intermediate product $S_{1}$ is necessary for the growth of species $X_{1}$. Hypothesis A3 means that the growth rate of species $X_{0}$ increases with the substrate $S_{0}$ but it is self-inhibited by the intermediate product $S_{1}$. Hypothesis A4 means that the growth of species $X_{1}$ increases with intermediate product $S_{1}$ produced by species $X_{0}$. Note that this defines a syntrophic relationship between the two species.

To ease the mathematical analysis of the system, we can rescale system (7) using the following change of variables adapted from [19]:

$$
s_{0}=Y_{2} S_{0}, \quad x_{0}=\frac{Y_{2}}{Y_{0}} X_{0}, \quad s_{1}=S_{1}, \quad x_{1}=\frac{1}{Y_{1}} X_{1},
$$

We obtain the following system

$$
\left\{\begin{aligned}
\frac{d s_{0}}{d t} & =D\left(s_{0}^{i n}-s_{0}\right)-\mu_{0}\left(s_{0}, s_{1}\right) x_{0} \\
\frac{d x_{0}}{d t} & =-D x_{0}+\mu_{0}\left(s_{0}, s_{1}\right) x_{0}-a_{0} x_{0} \\
\frac{d s_{1}}{d t} & =-D s_{1}+\mu_{0}\left(s_{0}, s_{1}\right) x_{0}-\mu_{1}\left(s_{1}\right) x_{1} \\
\frac{d x_{1}}{d t} & =-D x_{1}+\mu_{1}\left(s_{1}\right) x_{1}-a_{1} x_{1}
\end{aligned}\right.
$$

where $s_{0}^{i n}=Y_{2} S_{0}^{i n}$ and $\mu_{0}$ and $\mu_{1}$ are defined by

$\mu_{0}\left(s_{0}, s_{1}\right)=Y_{0} f_{0}\left(\frac{1}{Y_{2}} s_{0}, s_{1}\right) \quad$ and $\quad \mu_{1}\left(s_{2}\right)=Y_{1} f_{1}\left(s_{1}\right)$

The functions $\mu_{0}$ and $\mu_{1}$ are general functions with their own properties. Since 223 the functions $f_{0}$ and $f_{1}$ satisfy hypotheses $\mathbf{A 1}-\mathbf{A} \mathbf{4}$, it follows from (10) that functions $\mu_{0}$ and $\mu_{1}$ satisfy:

H1 For all $s_{0}>0$ and $s_{1} \geq 0, \mu_{0}\left(s_{0}, s_{1}\right)>0$ and $\mu_{0}\left(0, s_{1}\right)=0$. 
H2 For all $s_{1}>0, \mu_{1}\left(S_{1}\right)>0$ and $\mu_{1}(0)=0$.

227 H3 For all $s_{0}>0$ and $s_{1}>0, \frac{\partial \mu_{0}}{\partial s_{0}}\left(s_{0}, s_{1}\right)>0$ and $\frac{\partial \mu_{0}}{\partial s_{1}}\left(s_{0}, s_{1}\right)<0$.

${ }_{228} \quad$ H4 For all $s_{1}>0, \frac{d \mu_{1}}{d s_{1}}\left(s_{1}\right)>0$.

It should be noticed that (9) was studied in $[10,19]$ in the case where main-

\section{Steady-state and stability analysis}

A steady-state of (9) is a solution of the following nonlinear algebraic system obtained from (9) by setting the right-hand sides equal to zero:

$$
\begin{aligned}
D\left(s_{0}^{i n}-s_{0}\right)-\mu_{0}\left(s_{0}, s_{1}\right) x_{0} & =0 \\
-D x_{0}+\mu_{0}\left(s_{0}, s_{1}\right) x_{0}-a_{0} x_{0} & =0 \\
-D s_{1}+\mu_{0}\left(s_{0}, s_{1}\right) x_{0}-\mu_{1}\left(s_{1}\right) x_{1} & =0 \\
-D x_{1}+\mu_{1}\left(s_{1}\right) x_{1}-a_{1} x_{1} & =0
\end{aligned}
$$

A steady-state exists (or is said to be 'meaningful' [31]) if and only if all its components are non-negative. From equation (12) we deduce that:

$$
x_{0}=0 \quad \text { or } \quad \mu_{0}\left(s_{0}, s_{1}\right)=D+a_{0}
$$

and from equation (14) we deduce that:

$$
x_{1}=0 \quad \text { or } \quad \mu_{1}\left(s_{1}\right)=D+a_{1}
$$

The case $x_{0}=0$ and $x_{1}>0$ is excluded. Indeed, as a consequence of (16), we have $\mu_{1}\left(s_{1}\right)=D+a_{1}$ and, as a consequence of (13), we have $D s_{1}+\left(D+a_{1}\right) x_{1}=$ 0 , which is impossible since $s_{1} \geq 0$ and $x_{1}>0$. Therefore, three cases must be distinguished: 


\begin{tabular}{lll}
\hline Steady-state & Existence condition & Stability condition \\
\hline SS0 & Always exists & $s_{0}^{i n}<F_{0}(D)$ \\
SS1 & $s_{0}^{i n}>F_{0}(D)$ & $s_{0}^{i n}<F_{1}(D)$ \\
SS2 & $s_{0}^{i n}>F_{1}(D)$ & Always Stable \\
\hline
\end{tabular}

Table 1: Existence and local stability of steady-states.

SS0: $x_{0}=0, x_{1}=0$ where both species are washed out.

SS1: $x_{0}>0, x_{1}=0$, where species $x_{1}$ is washed out while $x_{0}$ survives.

SS2: $x_{0}>0, x_{1}>0$, where both species survive.

For the description of the steady-states and their stability, we need the following notations. Since the function $s_{1} \mapsto \mu_{1}\left(s_{1}\right)$ is increasing, it has an inverse function $y \mapsto M_{1}(y)$, so that, for all $s_{1} \geq 0$ and $y \in\left[0, \sup \mu_{1}(\cdot)\right)$

$$
s_{1}=M_{1}(y) \Longleftrightarrow y=\mu_{1}\left(s_{1}\right)
$$

Let $s_{1}$ be fixed. Since the function $s_{0} \mapsto \mu_{0}\left(s_{0}, s_{1}\right)$ is increasing, it has an inverse function $y \mapsto M_{0}\left(y, s_{1}\right)$, so that, for all $s_{0}, s_{1} \geq 0$, and $y \in\left[0, \sup \mu_{0}\left(\cdot, s_{1}\right)\right)$

$$
s_{0}=M_{0}\left(y, s_{1}\right) \Longleftrightarrow y=\mu_{0}\left(s_{0}, s_{1}\right)
$$

The inverse functions $s_{1}=M_{1}(y)$ and $s_{0}=M_{0}\left(y, s_{1}\right)$ can be calculated explicitly in the case of the Monod growth functions (23) considered in Section 6, see formulas $(25,(26))$. We define the functions:

$$
\begin{aligned}
& F_{0}(D)=M_{0}\left(D+a_{0}, 0\right) \\
& F_{1}(D)=M_{1}\left(D+a_{1}\right)+M_{0}\left(D+a_{0}, M_{1}\left(D+a_{1}\right)\right)
\end{aligned}
$$

Notice that $F_{1}(D)>F_{0}(D)$ for all $D \geq 0$, as long as they are both defined with the exception $F_{1}(0)=F_{0}(0)$, which holds if and only if $a_{0}=a_{1}=0$. Now, we can describe the steady-states of (9).

Proposition 1. Assume that assumptions H1-H4 hold. Then (9) has at most three steady-states:

- $S S O=\left(s_{0}=s_{0}^{i n}, x_{0}=0, s_{1}=0, x_{1}=0\right)$

It always exists. It is stable if and only if $s_{0}^{i n}<F_{0}(D)$. 
- $S S 1=\left(s_{0}, x_{0}=\frac{D}{D+a_{0}}\left(s_{0}^{i n}-s_{0}\right), s_{1}=s_{0}^{i n}-s_{0}, x_{1}=0\right)$ where $s_{0}$ is the solution of equation $\mu_{0}\left(s_{0}, s_{0}^{i n}-s_{0}\right)=D+a$. It exists if and only if $s_{0}^{i n}>F_{0}(D)$. It is stable if and only if $s_{0}^{i n}<F_{1}(D)$.

- $S S_{2}=\left(s_{0}, x_{0}=\frac{D}{D+a_{0}}\left(s_{0}^{i n}-s_{0}\right), s_{1}, x_{1}=\frac{D}{D+a_{1}}\left(s_{0}^{i n}-s_{0}-s_{1}\right)\right)$ where $s_{1}=M_{1}\left(D+a_{1}\right)$ and $s_{0}=M_{0}\left(D+a_{0}, M_{1}\left(D+a_{1}\right)\right)$. It exists if and only if $s_{0}^{i n}>F_{1}(D)$. It is stable if it exists.

The proof is given in the Appendix.

Notice that SS1 exists as soon as SS0 becomes unstable and SS2 exists as soon as SS1 becomes unstable. One concludes that for any value of the operating parameters, there is always one, and only one, steady-state which is stable. The results are summarized in Table 1. When decay effects are not taken into account, i.e. $a_{0}=a_{1}=0$, the system can be reduced to a planar system and global stability results can be obtained [10, 19]: for any pair of operating parameters, there is always one, and only one, steady-state which is globally asymptotically stable.

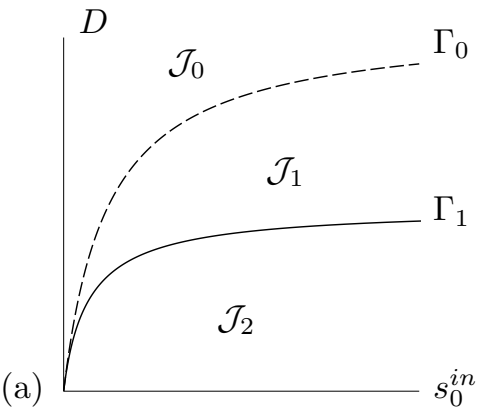

(b)

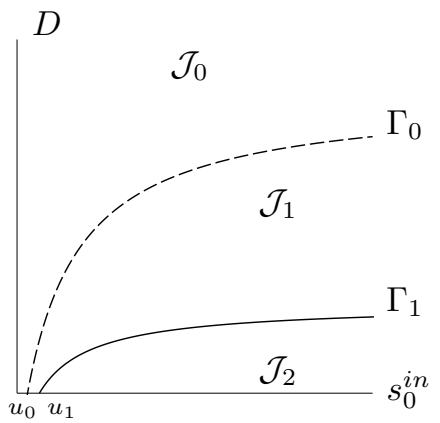

Figure 1: Operating diagram without (a) and with (b) maintenance effects. The values $u_{0}$ and $u_{1}$ are defined by (20)

\section{Operating diagram}

The operating diagram shows how the system behaves when we vary the two 


\begin{tabular}{ccccc}
\hline Condition & Region & SS0 & SS1 & SS2 \\
\hline$s_{0}^{i n}<F_{0}(D)$ & $\left(s_{0}^{i n}, D\right) \in \mathcal{J}_{0}$ & $\mathrm{~S}$ & & \\
$F_{0}(D)<s_{0}^{i n}<F_{1}(D)$ & $\left(s_{0}^{i n}, D\right) \in \mathcal{J}_{1}$ & $\mathrm{U}$ & $\mathrm{S}$ & \\
$F_{1}(D)<s_{0}^{i n}$ & $\left(s_{0}^{i n}, D\right) \in \mathcal{J}_{2}$ & $\mathrm{U}$ & $\mathrm{U}$ & $\mathrm{S}$ \\
\hline
\end{tabular}

Table 2: Existence and local stability of steady-states. The letter S (resp. U) means stable (resp. unstable). No letter means that the steady-state does not exist.

by (19). The curve $\Gamma_{0}$ of equation $s_{0}^{i n}=F_{0}(D)$ is the border which makes SS0 unstable and at the same time SS1 exists (the dashed curve in Fig. 1). The curve $\Gamma_{1}$ of equation $s_{0}^{i n}=F_{1}(D)$ is the border which makes SS1 unstable and at the same time SS2 exists (the solid curve in Fig. 1).

The curves $\Gamma_{0}$ and $\Gamma_{1}$ separate the operating plane $\left(s_{0}^{i n}, D\right)$ in three regions, as shown in Fig. 1, labelled $\mathcal{J}_{0}, \mathcal{J}_{1}$ and $\mathcal{J}_{2}$. The results of Prop. 1 are summarized in Table 2 which shows the existence and stability of the steady-states SS0, SS1 and SS2 in the regions $\mathcal{J}_{0}, \mathcal{J}_{1}$ and $\mathcal{J}_{2}$ of the operating diagram.

The values $u_{0}$ and $u_{1}$ plotted on the figure are obtained as follows:

$$
u_{0}=F_{0}(0)=M_{0}\left(a_{0}, 0\right), u_{1}=F_{1}(0)=M_{1}\left(a_{1}\right)+M_{0}\left(a_{0}, M_{1}\left(a_{1}\right)\right),
$$

If $a_{0} \geq \sup _{s_{0}>0} \mu_{0}\left(s_{0}, 0\right), F_{0}(0)$ is not defined and we let $u_{0}=+\infty$. In this case the regions $\mathcal{J}_{1}$ and $\mathcal{J}_{2}$ are empty. If $a_{1}<\sup _{s_{1}>0} \mu_{1}\left(s_{1}\right)$ or $a_{0} \geq \sup _{s_{0}>0} \mu_{0}\left(s_{0}-\right.$ $\left.M_{1}\left(a_{1}\right), M_{1}\left(a_{1}\right)\right), F_{1}(0)$ is not defined and we let $u_{1}=+\infty$. In this case the region $\mathcal{J}_{2}$ is empty. When maintenance effects are not taken into consideration, then $u_{0}=u_{1}=0$ and we have

$$
F_{0}(D)=M_{0}(D, 0), \quad F_{1}(D)=M_{1}(D)+M_{0}\left(D, M_{1}(D)\right)
$$

\section{A two-tiered microbial 'food chain'}

The model considered in [31] involves a two-tiered microbial 'food chain' with feedback inhibition, consisting of a propionate degrader and a hydrogenotrophic 
methanogen. The propionate degrader produces hydrogen which inhibits its own growth. Using the notations of ADM1 the model can be written as

$$
\left\{\begin{array}{l}
\frac{d S_{\text {pro }}}{d t}=D\left(S_{\text {pro }, \text { in }}-S_{\text {pro }}\right)-f_{0}\left(S_{\text {pro }}, S_{H_{2}}\right) X_{\text {pro }} \\
\frac{d X_{\text {pro }}}{d t}=-D X_{\text {pro }}+Y_{\text {pro }} f_{0}\left(S_{\text {pro }}, S_{H_{2}}\right) X_{\text {pro }}-k_{\text {dec }, \text { pro }} X_{\text {pro }} \\
\frac{d S_{H_{2}}}{d t}=-D S_{H_{2}}+\beta\left(1-Y_{\text {pro }}\right) f_{0}\left(S_{\text {pro }}, S_{H_{2}}\right) X_{\text {pro }}-f_{1}\left(S_{H_{2}}\right) X_{H_{2}} \\
\frac{d X_{H_{2}}}{d t}=-D X_{H_{2}}+Y_{H_{2}} f_{1}\left(S_{H_{2}}\right) X_{H_{2}}-k_{d e c, H_{2}} X_{H_{2}}
\end{array}\right.
$$

where $S_{\text {pro }}$ and $X_{\text {pro }}$ are propionate substrate and biomass concentrations; $S_{H_{2}}$ and $X_{H_{2}}$ are those for hydrogen; $Y_{\text {pro }}$ and $Y_{H_{2}}$ are yield coefficients and $\beta=0.43$ represents the part which goes to hydrogen substrate. The rest $(1-\beta=0.57)$ goes to acetate which is left out in the model. Both growth functions take Monod form with an hydrogen inhibition for the first one

$$
f_{0}\left(S_{\text {pro }}, S_{H_{2}}\right)=\frac{k_{m, p r o} S_{\text {pro }}}{K_{s, \text { pro }}+S_{\text {pro }}} \frac{1}{1+\frac{S_{H_{2}}}{K_{I, H_{2}}}}, \quad f_{1}\left(S_{H_{2}}\right)=\frac{k_{m, H_{2}} S_{H_{2}}}{K_{s, H_{2}}+S_{H_{2}}}
$$

Here, apart from the two operating (or control) parameters, which are the inflowing propionate concentration $S_{\text {pro,in }}$ and the dilution rate $D$, that can vary, all others have biological meaning and are fixed depending on the organisms and substrate considered [see 31, Table 1]. The aim of Xu et al. [31] was to study the stability of the steady-states of the model $(21,22)$ while varying the two operating (or control) parameters $D$ and $S_{\text {pro,in }}$. The system $(21,22)$ can have at most three steady-states: a trivial solution where both populations are washed out (SS0), a solution where $X_{H_{2}}$ is washed out while $X_{\text {pro }}$ survives (SS1) and a positive solution where both populations survive (SS2). The local stability of each steady-state was tested by linearisation around the steady-state values of the variables.

The basic results of the analysis of [31] are: for any pair of values of operating parameters, at most one steady-state is stable. When one of the decay terms is not taken into account, i.e. $k_{d e c, p r o}=0$ or $k_{d e c, H_{2}}=0$ in (21), there is always one and only one steady-state which is stable and SS2 is stable as long as it exists. When both decay effects are present, i.e. $k_{d e c, p r o}>0$ and $k_{d e c, \mathrm{H}_{2}}>0$ 

in (21), the authors were not able to check all the Routh-Hurwitz criteria for SS2. They claimed that SS2 is not necessarily stable in theory when it exists and they established numerically that with the ADM1 parameters values, SS2 to the questions asked by [31] in their paper.

More precisely, using the following simplified notations in (21)

$$
\begin{gathered}
S_{0}=S_{\text {pro }}, \quad S_{0}^{\text {in }}=S_{\text {pro }, \text { in }}, \quad S_{1}=S_{H_{2}}, \quad X_{0}=X_{\text {pro }}, \quad X_{1}=X_{H_{2}} \\
Y_{0}=Y_{\text {pro }}, \quad Y_{1}=Y_{H_{2}}, \quad Y_{2}=0.43\left(1-Y_{\text {pro }}\right), \quad a_{0}=k_{d e c, p r o}, \quad a_{1}=k_{d e c}, H_{2}
\end{gathered}
$$
and using the rescaling (10) and the biological parameters in (22) we obtain the

$$
\mu_{0}\left(s_{0}, s_{1}\right)=\frac{m_{0} s_{0}}{K_{0}+s_{0}} \frac{1}{1+s_{1} / K_{i}}, \quad \mu_{1}\left(s_{1}\right)=\frac{m_{1} s_{1}}{K_{1}+s_{1}}
$$

$$
\begin{array}{ll}
m_{0}=Y_{0} k_{m, p r o}, & K_{0}=Y_{2} K_{s, p r o}, \quad K_{i}=K_{I, H_{2}} \\
m_{1}=Y_{1} k_{m, H_{2}}, & K_{1}=K_{s, H_{2}}
\end{array}
$$

Let us describe our results in the particular case $(9,23)$. Notice that the growth functions (23) satisfy Assumptions H1-H4, so that Proposition 1 holds. In this case the inverse functions $M_{1}(y)$ and $y \mapsto M_{0}\left(y, s_{1}\right)$ of the functions $\mu_{1}\left(s_{1}\right)$ and $s_{0} \mapsto \mu_{0}\left(s_{0}, s_{1}\right)$ can be calculated explicitly: we have

$$
\begin{aligned}
& y \in\left[0, m_{1}\right) \mapsto M_{1}(y)=\frac{K_{1} y}{m_{1}-y}, \\
& y \in\left[0, \frac{m_{1}}{1+s_{1} / K_{i}}\right) \mapsto M_{0}\left(y, s_{1}\right)=\frac{K_{0} y}{\frac{m_{0}}{1+s_{1} / K_{i}}-y}
\end{aligned}
$$

Therefore, the functions $F_{1}(D)$ and $F_{2}(D)$ defined by (19) are given explicitly 
343 by

344

$$
\begin{aligned}
& F_{0}(D)=\frac{K_{0}\left(D+a_{0}\right)}{m_{1}-D-a_{0}} \\
& F_{1}(D)=\frac{K_{1}\left(D+a_{1}\right)}{m_{1}-D-a_{1}}+\frac{K_{0}\left(D+a_{0}\right)}{\frac{m_{0}}{1+\frac{K_{1}\left(D+a_{1}\right)}{\left(m_{1}-D-a_{1}\right) K_{i}}}-D-a_{0}}
\end{aligned}
$$

Notice that $F_{0}$ is defined on $\left[0, m_{1}-a_{0}\right)$ and $F_{1}$ is defined on $\left[0, D^{+}\right)$with $D^{+}<$ $m_{1}-a_{0}$. On the other hand, the solution $s_{0}$ of equation $\mu_{0}\left(s_{0}, s_{0}^{i n}-s_{0}\right)=D+a_{0}$, which is used in SS1, is simply the positive solution of the quadratic equation:

$$
m_{0} s_{0}=\left(D+a_{0}\right)\left(K_{0}+s_{0}\right)\left(1+\frac{s_{0}^{i n}-s_{0}}{K_{i}}\right)
$$

As a corollary of Proposition 1 we have the following result.

Proposition 2. Assume that $\mu_{0}$ and $\mu_{1}$ are given by (23). Let $F_{0}(D)$ and $F_{1}(D)$ be defined by (27). Then (9) has at most three steady-states

- $S S O=\left(s_{0}=s_{0}^{i n}, x_{0}=0, s_{1}=0, x_{1}=0\right)$ It always exists. It is stable if and only if $s_{0}^{i n}<F_{0}(D)$.

- $S S 1=\left(s_{0}, x_{0}=\frac{D}{D+a_{0}}\left(s_{0}^{i n}-s_{0}\right), s_{1}=s_{0}^{i n}-s_{0}, x_{1}=0\right)$ where $s_{0}$ is the positive solution of the quadratic equation (28). It exists if and only if $s_{0}^{\text {in }}>F_{0}(D)$. If it exists then it is stable if and only if $s_{0}^{\text {in }}<F_{1}(D)$.

- $S S 2=\left(s_{0}, x_{0}=\frac{D}{D+a_{0}}\left(s_{0}^{i n}-s_{0}\right), s_{1}, x_{1}=\frac{D}{D+a_{1}}\left(s_{0}^{i n}-s_{0}-s_{1}\right)\right)$ where

$$
s_{1}=\frac{K_{1}\left(D+a_{1}\right)}{m_{1}-D-a_{1}}, \quad s_{0}=\frac{K_{0}\left(D+a_{0}\right)}{\frac{m_{0}}{1+\frac{s_{1}}{K_{i}}}-D-a_{0}}
$$

It exists if and only if $s_{0}^{i n}>F_{1}(D)$. It is stable if it exists.

As a consequence of this result we obtain the results of [31], where the stability of $S S 2$ was proved only for a particular set of parameter values. To make the comparison possible the reader is advised on the main difference between our approach and [31]: we use the rescaling (10) and hence work with the growth 

functions (23), while Xu et al. [31] use a dimensionless rescaling. Despite this difference, both approaches are equivalent and hence must give the same results. Our quadratic equation (28) used in the description of SS1 is the same as their quadratic equation (A.1), or the quadratic equation without numbering preceding equation (B.1) [see 31, Appendix A and B].

\begin{tabular}{lll}
\hline Parameters & Units & Nominal Value \\
\hline$m_{0}$ & $\mathrm{~d}^{-1}$ & 0.52 \\
$K_{0}$ & $\mathrm{~kg} \mathrm{COD} / \mathrm{m}^{3}$ & 0.124 \\
$m_{1}$ & $\mathrm{~d}^{-1}$ & 2.10 \\
$K_{1}$ & $\mathrm{~kg} \mathrm{COD} / \mathrm{m}^{3}$ & $2.510^{-5}$ \\
$K_{i}$ & $\mathrm{~kg} \mathrm{COD} / \mathrm{m}^{3}$ & $3.510^{-6}$ \\
$a_{0}$ & $\mathrm{~d}^{-1}$ & 0.02 \\
$a_{1}$ & $\mathrm{~d}^{-1}$ & 0.02 \\
\hline
\end{tabular}

Table 3: Nominal parameters values.

(a)

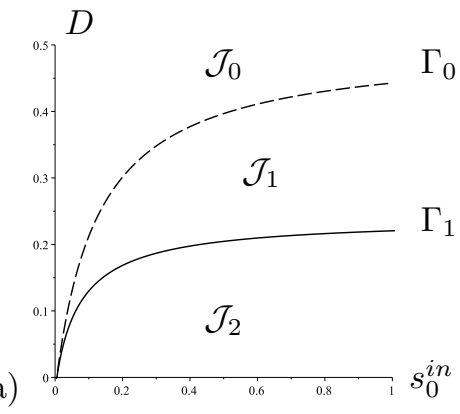

(b)

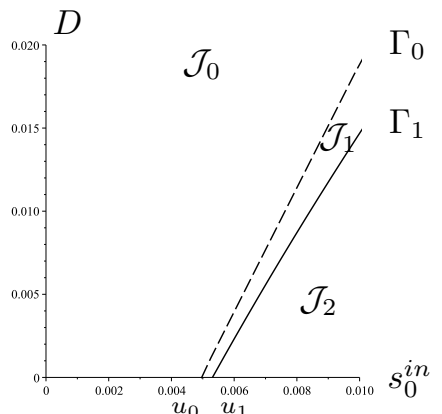

Figure 2: Operating diagram of the model (9)-(23). (a) The model was parametrised with the ADM1 consensus values listed in Table 3. (b) A magnification showing the values $u_{0}=$ $4.9610^{-3}, u_{1}=5.3110^{-3}$ defined by $(20)$.

For the numerical simulations we will use the nominal values of Table 3 obtained from Table 1 of [31] by using the formulas (24) and $a_{0}=k_{\text {dec,pro }}$, $a_{1}=k_{d e c, H_{2}}$. For these values of the parameters, the values $u_{0}$ and $u_{1}$ are very small, see Fig. 2. Notice that the scaling on the two coordinates in Fig. 2 are 
different from those of Fig. 2 of [31], since these authors used another rescaling.

\section{Discussion}

Following [31], we considered a two-tiered 'food chain' with feedback inhibition, which is a generalized model describing the syntrophic interaction of a propionate degrader and a hydrogenotropic methanogen. In the absence of maintenance these authors proved that this two-tiered 'food chain' is always stable. When maintenance is included in the model they were not able to check the Routh-Hurwitz criteria, and since the possibility of having at least one pair of complex eigenvalues with positive real parts is not theoretically excluded, they concluded that Hopf bifurcation can originate from SS2 [see 31, Appendix B]. However, using the consensus parameters of ADM1 and numerical simulations, they have shown that the model of the methanogenic two-tiered propionatehydrogen food chain is always stable [see 31, Section 6.2]. In this work we have generalized the model of the two-tiered 'food chain' of [31] by considering generic growth functions and we established the stability of the generalized model with maintenance terms.

In [31], the authors point out that introducing decay or maintenance in the classical predator-prey models results in instability and chaos [13]. For more details on food-chains in the chemostat the reader may consult [6, 24, 25]. Therefore, they observed that, in spite of the fundamental differences between their 'food chain' and the classical predator-prey models, the same intrinsic effect of maintenance on the stability of the food chain is observed [see 31, Section 7]: When maintenance is included in its description, the two-tiered generalized 'food chain' is not necessarily stable in theory. The results obtained in the present paper indicate that the two-tiered generalized 'food chain' is always stable, so that it is fundamentally different from the classical predatorprey model.

It should be noticed that the rescaling used by [31] gives a dimensionless model. However, our present rescaling (10) does not give a dimensionless model. 
The new variables $s_{0}, x_{0}, s_{1}$ and $x_{1}$ have the same dimensions as the original variables $S_{0}, X_{0}, S_{1}$ and $X_{1}$. The original growth functions (22) are transformed by our rescaling (10) in the growth functions (23) with the same scale imbalance in the half-saturation rates, see Table 3 . We cannot benefit from the dimensionless rescaling used by $\mathrm{Xu}$ et al. [31], because this rescaling uses some kinetics parameters of the specific growth functions (22) while we work with general unspecified growth functions. The benefit of our rescaling (10) is that it permits to fix the constant yields parameters $Y_{0}, Y_{1}$ and $Y_{2}$ in (7) to 1 , as shown by the rescaled model (9).

We were successful in checking the Routh-Hurwitz criteria because we work with general growth functions (defined by their qualitative properties given in assumptions A1-A4) and our computations are not encumbered by the specific form of the growth functions considered by Xu et al. [31]. These authors noticed [see 31, Section 7] that direct application of symbolic analysis programs, such as Maple or Mathematica, did not provide adequate solutions for the stability of the system. Actually we used the symbolic analysis program Maple to verify that the coefficients $\beta_{i}$ in the expression of the term $f_{1} f_{2} f_{3}-f_{1}^{2} f_{4}-f_{3}^{2}$ given in Appendix D are correct. It should be noticed that [31] have claimed [see 31, Remark 1] that their method is still effective for other growth functions. Our main contribution was to believe them and to try to solve the problem with general growth functions.

In the model (7) considered in this work, the first species $X_{0}$ uses the substrate $S_{0}$ for its growth and produces a substrate $S_{1}$ consumed by the second species $X_{1}$ for its growth. The substrate $S_{1}$ produced by the first species inhibits its own growth, that is, the growth function $f_{0}\left(S_{0}, S_{1}\right)$ is decreasing with respect to $S_{1}$. In practice, and in many complex models as the ADM1, it happens that the second species is also inhibited by the first substrate. Thus, it is interesting to consider the case where the second species is inhibited by the substrate $S_{0}$, namely that $f_{1}\left(S_{0}, S_{1}\right)$ also depends on $S_{0}$ and is decreasing with respect to $S_{0}$. It has been shown by Sari et al. [19] that the introduction of this last inhibiting relationship in the model completely changes the model proper- 
$J 48=\left[\begin{array}{cccc}-D-E x_{0} & -\mu_{0} & F x_{0} & 0 \\ E x_{0} & \mu_{0}-D-a_{0} & -F x_{0} & 0 \\ E x_{0} & \mu_{0} & -D-F x_{0}-G x_{1} & -\mu_{1} \\ 0 & 0 & G x_{1} & \mu_{1}-D-a_{1}\end{array}\right]$

ties while maintenance was not considered. In particular, the modified model exhibits multiplicity of positive steady-states. However, it should be stressed that these results were very general: whether this instability occurs for realistic environmental conditions or not is under investigation.

Another interesting question, which is the object of a future work, is to consider an input term $S_{1}^{i n}$ in (7), as well as a coefficient $\alpha<1$ in the dilution rate of the biomass, as it was the case in the general setting of (1). For instance if $S_{1}^{i n}>0$ then there exists an additional steady-state where $X_{0}=0$ is washed out and $X_{1}>0$ does not go to extinction.

where

$$
E=\frac{\partial \mu_{0}}{\partial s_{0}}\left(s_{0}, s_{1}\right)>0, \quad F=-\frac{\partial \mu_{0}}{\partial s_{1}}\left(s_{0}, s_{1}\right)>0, \quad G=\frac{d \mu_{1}}{d s_{1}}\left(s_{1}\right)>0
$$

${ }_{449}$ The eigenvalues of $J$ are the roots of its characteristic polynomial $\operatorname{det}(J-\lambda I)$.

450 Notice that we have used the opposite sign for the partial derivative $F=$ $451-\frac{\partial \mu_{0}}{\partial s_{1}}\left(s_{0}, s_{1}\right)$, so that all constants involved in the computations become posi-

452 tive, which will simplify the analysis of the characteristic polynomial of $J$. 


\section{Appendix B. SS0}

At SS0, $x_{0}=0, x_{1}=0$. As a result of (11) and (13), $s_{0}=s_{0}^{i n}$ and $s_{1}=0$. SS0 always exists. Evaluated at SS0, the Jacobian matrix (A.1) becomes

$$
J=\left[\begin{array}{cccc}
-D & -\mu_{0}\left(s_{0}^{i n}, 0\right) & 0 & 0 \\
0 & \mu_{0}\left(s_{0}^{i n}, 0\right)-D-a_{0} & 0 & 0 \\
0 & \mu_{0}\left(s_{0}^{i n}, 0\right) & -D & 0 \\
0 & 0 & 0 & -D-a_{1}
\end{array}\right]
$$

${ }_{454}$ Its eigenvalues are $\lambda_{1}=\mu_{0}\left(s_{0}^{i n}, 0\right)-D-a_{0}, \lambda_{2}=-D-a_{1}$ and $\lambda_{3}=\lambda_{4}=-D$.

${ }_{455}$ For being stable we need $\lambda_{1}<0$. Therefore SS0 is unstable if and only if

$$
\mu_{0}\left(s_{0}^{i n}, 0\right)>D+a_{0}
$$

Since the function $s_{0} \mapsto \mu_{0}\left(s_{0}, 0\right)$ is increasing, and using (18) we have the following equivalence

$$
\mu_{0}\left(s_{0}^{i n}, 0\right)>D+a_{0} \Longleftrightarrow s_{0}^{i n}>M_{0}\left(D+a_{0}, 0\right)
$$

${ }_{457}$ Therefore, according to (19), (B.1) is equivalent to $s_{0}^{i n}>F_{0}(D)$.

\section{${ }_{458}$ Appendix C. SS1}

At SS1, $x_{0} \neq 0, x_{1}=0$. As a consequence of $(15) \mu_{0}\left(s_{0}, s_{1}\right)=D+a_{0}$. As a result of (11) and (13)

$$
D\left(s_{0}^{i n}-s_{0}\right)=\mu_{0}\left(s_{0}, s_{1}\right) x_{0} \quad \text { and } \quad D s_{1}=\mu_{0}\left(s_{0}, s_{1}\right) x_{0}
$$

459 Hence $x_{0}=\frac{D}{D+a_{0}}\left(s_{0}^{i n}-s_{0}\right)$ and $D\left(s_{0}^{i n}-s_{0}\right)=D s_{1}$, so that $s_{0}+s_{1}=s_{0}^{i n}$.

${ }_{460}$ Therefore $s_{0}$ is a solution of equation

$$
\mu_{0}\left(s_{0}, s_{0}^{i n}-s_{0}\right)=D+a_{0}
$$

SS1 exists if and only if this equation has a solution in the interval $\left(0, s_{0}^{i n}\right)$. The function $s_{0} \mapsto \psi\left(s_{0}\right)=\mu_{0}\left(s_{0}, s_{0}^{i n}-s_{0}\right)$ is increasing since its derivative $\frac{d \psi}{d s_{0}}=\frac{\partial \mu_{0}}{\partial s_{0}}-\frac{\partial \mu_{0}}{\partial s_{1}}>$ is positive. Using $\psi(0)=0$ and $\psi\left(s_{0}^{i n}\right)=\mu\left(s_{0}^{i n}, 0\right)$ we 
conclude that equation (C.1) has a solution in the interval $\left(0, s_{0}^{i n}\right)$ if and only if $\psi\left(s_{0}^{i n}\right)=\mu\left(s_{0}^{i n}, 0\right)>D+a_{0}$, that is to say condition (B.1) holds. The condition of existence of SS1 is then equivalent to the condition of instability of SS0.

Evaluated at SS1, the Jacobian matrix (A.1) becomes:

$$
J=\left[\begin{array}{cccc}
-D-E x_{0} & -D-a_{0} & F x_{0} & 0 \\
E x_{0} & 0 & -F x_{0} & 0 \\
E x_{0} & D+a_{0} & -D-F x_{0} & -\mu_{1} \\
0 & 0 & 0 & \mu_{1}-D-a_{1}
\end{array}\right]
$$

Its characteristic polynomial is:

$\operatorname{det}(J-\lambda I)=\left(\lambda-\mu_{1}+D+a_{1}\right)(\lambda+D)\left(\lambda^{2}+\left[D+(E+F) x_{0}\right] \lambda+\left(D+a_{0}\right)(E+F) x_{0}\right)$

Its eigenvalues are $\lambda_{1}=\mu_{1}-D-a_{1}, \lambda_{2}=-D$ and $\lambda_{3}$ and $\lambda_{4}$ are the roots of the following quadratic equation:

$$
\lambda^{2}+\left[D+(E+F) x_{0}\right] \lambda+\left(D+a_{0}\right)(E+F) x_{0}=0
$$

${ }_{462}$ Since $\lambda_{3} \lambda_{4}=\left(D+a_{0}\right)(E+F) x_{0}>0$ and $\lambda_{3}+\lambda_{4}=-\left[D+(E+F) x_{0}\right]<0$,

${ }_{463}$ the real parts of $\lambda_{3}$ and $\lambda_{4}$ are negative. So for being stable it must be $\lambda_{1}<0$.

${ }_{464}$ Therefore SS1 is stable if and only if

$$
\mu_{1}\left(s_{0}^{i n}-s_{0}\right)<D+a_{1} \text {, where } s_{0} \text { is the solution of (C.1) }
$$

Since the function $s_{1} \mapsto \mu_{1}\left(s_{1}\right)$ is increasing, we have the following equivalence

$$
\mu_{1}\left(s_{0}^{i n}-s_{0}\right)<D+a_{1} \Longleftrightarrow s_{0}<s_{0}^{i n}-M_{1}\left(D+a_{1}\right)
$$

Since the function $s_{0} \mapsto \psi\left(s_{0}\right)=\mu_{0}\left(s_{0}, s_{0}^{i n}-s_{0}\right)$ is decreasing, we deduce that $\psi\left(s_{0}\right)>\psi\left(s_{0}^{i n}-M_{1}\left(D+a_{1}\right)\right)$. Since $s_{0}$ be the solution of (C.1),

$$
\psi\left(s_{0}\right)=\mu_{0}\left(s_{0}, s_{0}^{i n}-s_{0}\right)=D+a_{0}
$$

${ }_{466}$ Therefore, the condition (C.2) of stability of SS1 is equivalent to:

$$
D+a_{0}<\mu_{0}\left(s_{0}^{i n}-M_{1}\left(D+a_{1}\right), M_{1}\left(D+a_{1}\right)\right)
$$


Since the function $s_{0} \mapsto \mu_{0}\left(s_{0}, M_{1}\left(D+a_{1}\right)\right)$ is increasing, and using (18), the condition (C.3) is equivalent to

$$
s_{0}^{i n}-M_{1}\left(D+a_{1}\right)<M_{0}\left(D+a_{0}, M_{1}\left(D+a_{1}\right)\right)
$$

which is, according to (19), equivalent to

$$
s_{0}^{i n}<M_{1}\left(D+a_{1}\right)+M_{0}\left(D+a_{0}, M_{1}\left(D+a_{1}\right)\right)=: F_{1}(D)
$$

\section{${ }_{468}$ Appendix D. SS2}

At SS2, $x_{0} \neq 0, x_{1} \neq 0$. As a consequence of (15) and (16) $s_{0}$ and $s_{1}$ are solutions of the set of equations

$$
\mu_{0}\left(s_{0}, s_{1}\right)=D+a_{0}, \quad \mu_{1}\left(s_{1}\right)=D+a_{1}
$$

${ }_{469}$ Using (17) we obtain $s_{1}=M_{1}\left(D+a_{1}\right)$ and $s_{0}$ is a solution of equation

$$
\mu_{0}\left(s_{0}, M_{1}\left(D+a_{1}\right)\right)=D+a_{0}
$$

Using (18) we obtain $s_{0}=M_{0}\left(D+a_{0}, M_{1}\left(D+a_{1}\right)\right)$. As a result of (11) and

$$
x_{0}=\frac{D}{D+a_{0}}\left(s_{0}^{i n}-s_{0}\right), \quad x_{1}=\frac{D}{D+a_{1}}\left(s_{0}^{i n}-s_{0}-s_{1}\right)
$$

SS2 exists if and only if $s_{0}^{i n}>s_{0}+s_{1}$, that is

$$
s_{0}^{\text {in }}>M_{1}\left(D+a_{1}\right)+M_{0}\left(D+a_{0}, M_{1}\left(D+a_{1}\right)\right)=: F_{1}(D)
$$

Evaluated at SS2, the Jacobian matrix (A.1) becomes:

$$
J=\left[\begin{array}{cccc}
-D-E x_{0} & -D-a_{0} & F x_{0} & 0 \\
E x_{0} & 0 & -F x_{0} & 0 \\
E x_{0} & D+a_{0} & -D-F x_{0}-G x_{1} & -D-a_{1} \\
0 & 0 & G x_{1} & 0
\end{array}\right]
$$

Its characteristic polynomial is:

$$
\operatorname{det}(J-\lambda I)=\lambda^{4}+f_{1} \lambda^{3}+f_{2} \lambda^{2}+f_{3} \lambda+f_{4}
$$


where

$$
\begin{gathered}
f_{1}=G x_{1}+(E+F) x_{0}+2 D \\
f_{2}=E G x_{0} x_{1}+\left(2 D+a_{0}\right)(E+F) x_{0}+\left(2 D+a_{1}\right) G x_{1}+D^{2} \\
f_{3}=\left(2 D+a_{0}+a_{1}\right) E G x_{0} x_{1}+D\left(D+a_{0}\right)(E+F) x_{0}+D\left(D+a_{1}\right) G x_{1} \\
f_{4}=\left(D+a_{0}\right)\left(D+a_{1}\right) E G x_{0} x_{1}
\end{gathered}
$$

471 Hence

$$
f_{i}>0 \text { for } i=1 \cdots 4
$$

Since the quantity $E+F$ occurs so often in the computations, we use the notation $H=E+F$. Straightforward calculations show that:

$$
f_{1} f_{2}-f_{3}=2 D^{3}+\alpha_{2} D^{2}+\alpha_{1} D+\alpha_{0}
$$

where

$$
\begin{gathered}
\alpha_{2}=4\left(H x_{0}+G x_{1}\right) \\
\alpha_{1}=2\left(H x_{0}+G x_{1}\right)^{2}+a_{0} H x_{0}+a_{1} G x_{1} \\
\alpha_{0}=E G\left(H x_{0}+G x_{1}\right) x_{0} x_{1}+a_{0} H^{2} x_{0}^{2}+\left(a_{0}+a_{1}\right) F G x_{0} x_{1}+a_{1} G^{2} x_{1}^{2}
\end{gathered}
$$

Thus

$$
f_{1} f_{2}-f_{3}>0
$$

On the other hand we have

$$
f_{1} f_{2} f_{3}-f_{1}^{2} f_{4}-f_{3}^{2}=\beta_{5} D^{5}+\beta_{4} D^{4}+\beta_{3} D^{3}+\beta_{2} D^{2}+\beta_{1} D+\beta_{0}
$$

where

$$
\begin{gathered}
\beta_{5}=2\left(H x_{0}+G x_{1}\right) \\
\beta_{4}=4\left(H x_{0}+G x_{1}\right)^{2}+2 a_{0} H x_{0}+2 a_{1} G x_{1}
\end{gathered}
$$

$$
\begin{aligned}
\beta_{3}= & 2\left(H x_{0}+G x_{1}\right)^{3}+4 E G\left(H x_{0}+G x_{1}\right) x_{0} x_{1} \\
& +5 a_{0} H^{2} x_{0}^{2}+\left(a_{0}+a_{1}\right)(3 E+5 F) G x_{0} x_{1}+5 a_{1} G^{2} x_{1}^{2}
\end{aligned}
$$




$$
\begin{aligned}
\beta_{2}= & 4 E G\left(H x_{0}+G x_{1}\right)^{2} x_{0} x_{1} \\
& +3 a_{0} H^{3} x_{0}^{3}+\left(a_{0} E+2 a_{1} E+6 a_{0} H+3 a_{1} F\right) G H x_{0}^{2} x_{1} \\
& +\left(2 a_{0} E+a_{1} E+3 a_{0} F+6 a_{1} H\right) G^{2} x_{0} x_{1}^{2}+3 a_{1} G^{3} x_{1}^{3} \\
& +a_{0}^{2} F(F+2 E) x_{0}^{2}+\left(a_{0} E x_{0}-a_{1} G x_{1}\right)^{2}+2 a_{0} a_{1} G F x_{0} x_{1}
\end{aligned}
$$

$$
\begin{aligned}
\beta_{1}= & 2 E^{2} G^{2}\left(H x_{0}+G x_{1}\right) x_{0}^{2} x_{1}^{2}+\left(4 a_{0}+a_{1}\right) E G H^{2} x_{0}^{3} x_{1} \\
& +\left(a_{0}+a_{1}\right)(3 E+5 F) E G^{2} x_{0}^{2} x_{1}^{2}+\left(a_{0}+4 a_{1}\right) E G^{3} x_{0} x_{1}^{3} \\
& +a_{0}^{2}\left(3 E^{2}+3 E F+F^{2}\right) F x_{0}^{3}+a_{0}\left(2 a_{0} E+a_{0} F+2 a_{1} F\right) G F x_{0}^{2} x_{1} \\
& +\left(E x_{0}+G x_{1}\right)\left(a_{0} E x_{0}-a_{1} G x_{1}\right)^{2}+\left(2 a_{0} a_{1}+a_{1}^{2}\right) G^{2} F x_{0} x_{1}^{2}
\end{aligned}
$$

$$
\begin{aligned}
\beta_{0}= & \left(a_{0}+a_{1}\right) E^{2} G^{2}\left(H x_{0}+G x_{1}\right) x_{0}^{2} x_{1}^{2}+a_{0}^{2}(2 E+F) E F G x_{0}^{3} x_{1} \\
& +\left(a_{0}^{2}+a_{1}^{2}\right) E F G^{2} x_{0}^{2} x_{1}^{2}+\left(a_{0} E x_{0}-a_{1} G x_{1}\right)^{2} E G x_{0} x_{1}
\end{aligned}
$$

$$
f_{1} f_{2} f_{3}-f_{1}^{2} f_{4}-f_{3}^{2}>0
$$

${ }_{481}$ According to (D.2), (D.3) and (D.4) the Routh-Hurwitz criteria are satisfied.

482 Therefore, SS2 is stable as long as it exists.

\section{${ }_{483}$ Acknowledgements}

${ }_{484}$ This work was supported by the Treasure network. The authors warmly 485 thank Matthew Wade and the group Modemic for fruitful discussions and two ${ }_{486}$ anonymous referees for their comments and suggestions. This work was finalized 487 during the workshop Microbial ecology and mathematical modelling, organized ${ }_{488}$ in December 2014 at the Centre Interfacultaire Bernoulli (CIB) of the Ecole ${ }_{489}$ Polytechnique Fédérale de Lausanne (EPFL) in Switzerland, with funding of ${ }_{490}$ the Swiss National Science Foundation. 


\section{References}

[1] V. Alcaraz-Gonzalez, J. Harmand, A. Rapaport, J. P. Steyer, V. Gonzalez Alvarez, C. Pelayo Ortiz (2002). Software sensors for highly uncertain WWTPs : a new approach based on interval observers. Water Research 36, 2515-2524. doi:10.1016/S0043-1354(01)00466-3

[2] V. Alcaraz-Gonzalez, J. Harmand, A. Rapaport, J. P. Steyer, V. Gonzalez Alvarez, C. Pelayo Ortiz (2005). Application of a Robust Interval Observer to an Anaerobic Digestion Process. Developments in Chemical Engineering and Mineral Processing 13, 3-4. DOI: 10.1002/apj.5500130308

[3] D.J. Batstone, J. Keller, I. Angelidaki, S.V. Kalyuzhnyi, S.G. Pavlostathis, A. Rozzi, W.T.M Sanders, H. Siegrist, V.A. Vavilin (2002). The Iwa Anaerobic Digestion Model No 1 (ADM1). Water Sci. Technol. 45, 65-73.

[4] B. Benyahia, T. Sari, B. Cherki, J. Harmand (2012). Bifurcation and stability analysis of a two step model for monitoring anaerobic digestion processes. J. Process Control 22, 1008-1019. doi:10.1016/j.jprocont.2012.04.012

[5] O. Bernard, Z. Hadj-Sadock, D. Dochain, A. Genovesi, J.-P. Steyer (2001). Dynamical model development and parameter identification for an anaerobic wastewater treatment process. Biotechnol. Bioeng. 75, 424-438. DOI: 10.1002/bit.10036

[6] M.P. Boer, B.W. Kooi, S.A.L.M. Kooijman (1998). Food chain dynamics in the chemostat. Mathematical Biosciences 150, 43-62. doi:10.1016/S0025$5564(98) 00010-8$

[7] A. Bornhöft, R. Hanke-Rauschenbach, K. Sundmacher (2013). Steadystate analysis of the Anaerobic Digestion Model No. 1 (ADM1). Nonlinear Dynamics 73, 535-549. DOI 10.1007/s11071-013-0807-x

[8] A. Burchard (1994). Substrate degradation by a mutualistic association of two species in the chemostat. J. Math. Biol. 32, 465-489. DOI 10.1007/BF00160169 
[9] M. El Hajji, J. Harmand, H. Chaker and C. Lobry (2009). Association between competition and obligate mutualism in a chemostat, J. Biol. Dynamics, 3, 635-647. DOI:10.1080/17513750902915978

[10] M. El Hajji, F. Mazenc and J. Harmand (2010). A mathematical study of a syntrophic relationship of a model of anaerobic digestion process. Math. Biosci. Eng. 7, 641-656. doi: 10.3934/mbe.2010.7.641

[11] E. Harvey, J. Heys, T. Gedeon (2014). Quantifying the effects of the division of labor in metabolic pathways. J. Theoret. Biol. 360, 222-242. doi:10.1016/j.jtbi.2014.07.011

[12] IWA Task Group for Mathematical Modelling of Anaerobic Digestion Processes (2002). Anaerobic Digestion Model No.1 (ADM1). Scientific and Technical Report No. 13, IWA Publishing, London.

[13] B.W. Kooi, M.P. Boer (2003). Chaotic behaviour of a predator-prey system in the chemostat. Second International Conference on Dynamics of Continuous, Discrete and Impulsive Systems (London, ON, 2001). Dyn. Contin. Discrete Impuls. Syst. Ser. B Appl. Algorithms 10, 259-272.

[14] R. Kreikenbohm, E. Bohl (1986). A mathematical model of syntrophic cocultures in the chemostat. FEMS Microbiol. Ecol. 38: 131-140. doi:10.1016/0378-1097(86)90044-3

[15] R. Kreikenbohm, E. Bohl (1988). Bistability in the Chemostat. Ecological Modelling 43, 287-301. doi:10.1016/0304-3800(88)90009-9

[16] B.J. Ni, G.P. Sheng, Yu, H.Q. (2011). Model-based characterization of endogenous maintenance, cell death and predation processes of activated sludge in sequencing batch reactors. Chemical Engineering Science 66, 747754. doi:10.1016/j.ces.2010.11.033

[17] I. Ramirez, E.I.P. Volcke, R. Rajinikanth, J.P. Steyer (2009). Modelling microbial diversity in anaerobic digestion through an extended adm1 model. Water Res. 43, 2787-2800. doi:10.1016/j.watres.2009.03.034 
[18] P.J. Reilly (1974). Stability of commensalistic systems. Biotechnol. Bioeng. 16, 1373-1392. DOI: 10.1002/bit.260161006

[19] T. Sari, M. El Hajji, J. Harmand (2012). The mathematical analysis of a syntrophic relationship between two microbial species in a chemostat. Math. Biosci. Eng. 9, 627-645. doi:10.3934/mbe.2012.9.627

[20] T. Sari, J. Harmand (2014). Maintenance does not affect the stability of a two-tiered microbial 'food chain'. https://hal.archives-ouvertes.fr/hal01026149v1

[21] M. Sbarciog, M. Loccufier, E Noldus (2010). Determination of appropriate operating strategies for anaerobic digestion systems. Biochemical engineering journal 51 (3), 180-188. doi:10.1016/j.bej.2010.06.016

[22] G. Stephanopoulos (1981). The dynamics of commensalism. Biotechnol. Bioeng. 23, 2243-2255. DOI:10.1002/bit.260231008

[23] J.B. Van Lier, N.A. Mahmoud, G. Zeeman (2008). Anaerobic wastewater treatment. In: M. Henze, M.C.M. van Loodsdrecht, G.A. Ekema, D. Brdjanovic (Eds.), Biological Wastewater Treatment: Principle, Modelling and Design. IWA Publishing, London, 415-456.

[24] G.A.K. van Voorn, B.W. Kooi, M.P. Boer (2010). Ecological consequences of global bifurcations in some food chain models. Mathematical Biosciences 226, 120-133. doi:10.1016/j.mbs.2010.04.005

[25] D.V. Vayenas, S. Pavlou (1999). Chaotic dynamics of a food web in a chemostat. Mathematical Biosciences 162, 69-84. doi:10.1016/S00255564(99)00044-9

[26] E.I.P. Volcke, M. Sbarciog, E.J.L. Noldus, B. De Baets, M. Loccufier (2010). Steady-state multiplicity of two-step biological conversion systems with general kinetics. Mathematical Biosciences 228, 160-170. doi:10.1016/j.mbs.2010.09.004 
[27] M.J. Wade, J. Harmand, B. Benyahia, T. Bouchez, S. Chaillou, B. Cloez, J.-J. Godon, B. Moussa Boudjemaa, A. Rapaport, T. Sari, R. Arditi, C. Lobry (2016). Perspectives in mathematical modelling for microbial ecology. Ecological Modelling 321, 64-74. doi:10.1016/j.ecolmodel.2015.11.002

[28] M. Weedermann, G. Seo, G. Wolkowicz (2013). Mathematical Model of Anaerobic Digestion in a Chemostat: Effects of Syntrophy and Inhibition. Journal of Biological Dynamics 7, 59-85. DOI:10.1080/17513758.2012.755573

[29] M. Weedermann, G. Wolkowicz, J. Sasara (2015). Optimal biogas production in a model for anaerobic digestion. Nonlinear Dynamics 81, 1097-1112. DOI 10.1007/s11071-015-2051-z

[30] T. G. Wilkinson, H. H. Topiwala, G. Hamer (1974). Interactions in a Mixed Bacterial Population Growing on Methane in Continuous Culture. Biotechnol. Bioeng. 16, 41-59. DOI: 10.1002/bit.260160105

[31] A. Xu, J. Dolfing, T.P. Curtis, G. Montague, E. Martin (2011). Maintenance affects the stability of a two-tiered microbial 'food chain'? J. Theoret. Biol. 276, 35-41. doi:10.1016/j.jtbi.2011.01.026 